\title{
The Development and Precision of a Custom-Made Skitester
}

\author{
Teemu Lemmettylä ${ }^{1 *}$, Teemu Heikkinen $^{1}$, Olli Ohtonen ${ }^{1}$, Stefan Lindinger ${ }^{2}$ and \\ Vesa Linnamo ${ }^{1}$ \\ ${ }^{1}$ Sports Technology Unit, Faculty of Sport and Health Sciences, University of Jyväskylä, Vuokatti, Finland, ${ }^{2}$ Department of \\ Food and Nutrition and Sport Science, Center for Health and Performance, Faculty of Education, University of Gothenburg, \\ Gothenburg, Sweden
}

In the sport of cross-country skiing, equipment has a direct influence on results. Ski teams do extensive testing of different ski base grinds and products on a yearly basis. To achieve reliable results, the quality of methods used for testing skis needs to be taken in to account in addition to factors including the physical characteristics of testing personnel and changes in weather conditions. The aim of this study was to introduce a custom-made skitester, that was developed for testing skis on real snow, in laboratory conditions, and to evaluate its precision. The current skitester is capable of glide testing both classic and skate skis as well as kick simulation for the testing of grip waxes. In the present study, glide testing precision was completed in three different conditions. Velocity and pressure of skis were evaluated in three different temperature conditions. During kick simulation, precision was determined in one temperature condition. For glide testing,

Edited by: Igor Velkavrh,

V-Research GmbH, Austria

Reviewed by:

Erik Petrus Andersson,

Arctic University of Norway, Norway Yoshitaka Nakanishi, Kumamoto University, Japan

*Correspondence: Teemu Lemmettylä teemu.j.lemmettyla@jyu.fi

Specialty section: This article was submitted to Tribology,

a section of the journal

Frontiers in Mechanical Engineering

Received: 31 January 2021 Accepted: 13 April 2021 Published: 12 May 2021

Citation:

Lemmettylä T, Heikkinen T,

Ohtonen $O$, Lindinger $S$ and Linnamo V (2021) The Development and Precision of a Custom-Made Skitester.

Front. Mech. Eng. 7:661947. doi: 10.3389/fmech.2021.661947 the precision of the measurement unit was able to distinguish the differences between skis with a relative variation of $0.6-1.1 \%$. However, the track preparation process caused variation. For kick simulation, precision of the measurement unit was slightly higher (2.5\%), and track preparation caused less variation. The skitester is capable of distinguishing the differences between both skate and classic cross-country skis with certain limitations.

\section{Keywords: tribology, cross-country skiing, coefficient of friction, ski testing, snow, waxing}

\section{INTRODUCTION}

Equipment plays an important role in cross-country skiing because ski glide directly influences the finishing time of the skier (Moxnes et al., 2014; Pellegrini et al., 2018). To improve ski performance, national teams use a significant part of their budgets to develop and test methods to improve the performance of skis. The main objective of the ski service team in World Cup competitions is to find the best possible ski and ski base preparation to match the current environmental conditions.

\section{Ski Testing}

The base of a ski can be optimized for glide by manipulating its structure and surface. Machine and hand structures can be added to optimize glide in various different snow conditions (Moldestad, 1999; Breitschädel et al., 2010b). Waxing is typically completed in layers using a variety of products ranging from basic paraffin waxes to highly fluorinated products (Moldestad, 1999; Karlöf and Axell, 2005; Breitschädel et al., 2014). In competitions where the classic technique is used, the amount and type of grip wax must also be considered. Well-prepared classic skis enable skiers to ski more economically by enabling more efficient use of horizontal force production 
(Vähäsöyrinki et al., 2008; Linnamo et al., 2009b; Ainegren et al., 2014). Adding too much grip wax, however, increases gliding friction of the grip wax zone in both flat and downhill sections of the track (Stöggl et al., 2019), slowing the skier. The characteristics of skis used for testing have a direct impact on ski service quality. Ski service methods are most often developed through the extensive testing meaning that any potential for error during the testing ski identification process needs to be minimized. When the testing skis are properly identified, recognizing optimal combinations of ski base preparation including wax, grinds, and handmade structure is more reliable.

The complexity of ski testing can be observed at World Cup races where testing is completed outdoors on a ski track. As separate measurement or timing systems are not allowed (ICR Cross Country, 2020), ski testing is completed using subjective feelings of glide or by parallel gliding, in which two ski service members glide side-by-side on a downhill to determine which ski has better glide. This ski testing method gives an overall estimation of ski glide, however, the body mass of ski service member as well as their frontal area influences these results (Spring et al., 1988; Swarén et al., 2014). Several environmental factors may also change during ski testing ultimately influencing test results: wind speed and direction; air and snow temperature; snow deformation on the testing track (Nachbauer et al., 1996; Fauve et al., 2005).

\section{Ski Selection}

Typically, 4-8 pairs of similar skis are identified for a ski service testing pack. The similarity of testing skis is highly important because differences between base preparations are identified using these skis. In recent years testing ski selection has been aided by methods including measurement of ski stiffness and height (Bäckström et al., 2008; Breitschädel et al., 2010a), however, the hand selection of testing skis still plays a an important role. Hand selection of skis is highly influenced by the personal preferences of the individual making the selection and is based on identification of desired characteristics of the ski including the location and length of the pressure zones. Different criteria are used for selecting skate and classic skis. The length and height of the kick zone is relatively easy to identify from a classical ski and this information can be used to apply the appropriate amount of grip wax. In the assessment of skating skis, results give an overall estimation of ski characteristics that cannot be used to optimize waxing. The final selection of testing skis is always completed on real snow and traditionally, the only methods used for testing ski selection have been timing or parallel testing.

\section{Gliding on Snow}

Friction on snow is explained as mechanical friction lubricated with a liquid layer between the ski and snow. The relationship of the liquid layer thickness to the coefficient of the friction $(\mathrm{CoF})$ is parabolic where the most favorable gliding conditions are achieved at the optimal lubrication level and CoF increases if the lubrication level is higher or lower (Hamrock and Dowson, 1981). Factors influencing the liquid layer thickness under a moving ski have been examined from several perspectives. The velocity of the ski causing the frictional heating and the temperature are factors regulating the liquid layer thickness between ski and snow while pressure is reported to have less influence on the CoF. Mechanical friction is associated with snow type and hardness (Colbeck, 1994; Moldestad, 1999; Theile et al., 2009). In extensive use of the same ski track, frictional heating from the ski base can change the CoF. Frictional heating causes snow deformation when the track is skied over several times (Eriksson and Nupen, 1955). Mechanical phenomenon including vibrations of the ski are also reported to influence ski glide (Koptyug et al., 2012).

\section{Tribometers for Ski Testing}

The frictional characteristics of a ski base sliding over snow or ice have been studied with tribometers in lab conditions (Shimbo, 1961; Buhl et al., 2001; Bäurle, 2006; Theile et al., 2009). Some linear tribometers have been built that are capable of crosscountry ski glide testing (Buhl et al., 2001; Linnamo et al., 2009a; Fenre, 2016; Hasler et al., 2016). The current linear tribometer (skitester) was introduced in 2009 for simulating the classical skiing kick motion (Linnamo et al., 2009a). After that, several modifications have been made in order to improve measurement reliability and to control the environment in which tests are carried out. To the best of our knowledge, the current skitester is the only linear tribometer capable of simulating classical skiing kick.

Equipment testing in on the ski tracks during World Cups, World Championships, and the Olympics are limited to methods such as parallel gliding. Regrettably, these field methods only partially reveal the factors influencing results related to glide and kick. The quality of ski testing can be enhanced by excluding some of the environmental factors, by improving the testing protocols, and/or by improving the quality of the test skis by minimizing the differences between skis in the testing pack (Fauve et al., 2005). The aim of this paper is to introduce the technical aspects of the current skitester and to evaluate the precision of measurements in both glide and kick testing.

\section{MATERIALS AND METHODS}

\section{Skitester}

The skitester is located in a custom-made low temperature laboratory (Figure 1A). This low temperature laboratory was built from two insulated 12-m-long containers and it has two separate rooms. The smaller room serves as an entrance and a working space for computing and maintenance. The main room, where the skitester is located, is temperature controlled. A condensing unit (Bitzer LHV7V, Germany) is installed outside of the laboratory and cooled refrigerant is used to cool both laboratory air as well as the ski track. The room is cooled with an air cooler (Stefani SHCP 035/3, Italy). Under the ski track are custom made cooling pipes (Figure 3A). The air and track temperature cooling systems can be programmed separately to enable e.g., the simulation of changing conditions. For example, the track can be programed to temperatures ranging from -20 to $+10^{\circ} \mathrm{C}$ while air temperature can be programmed to 


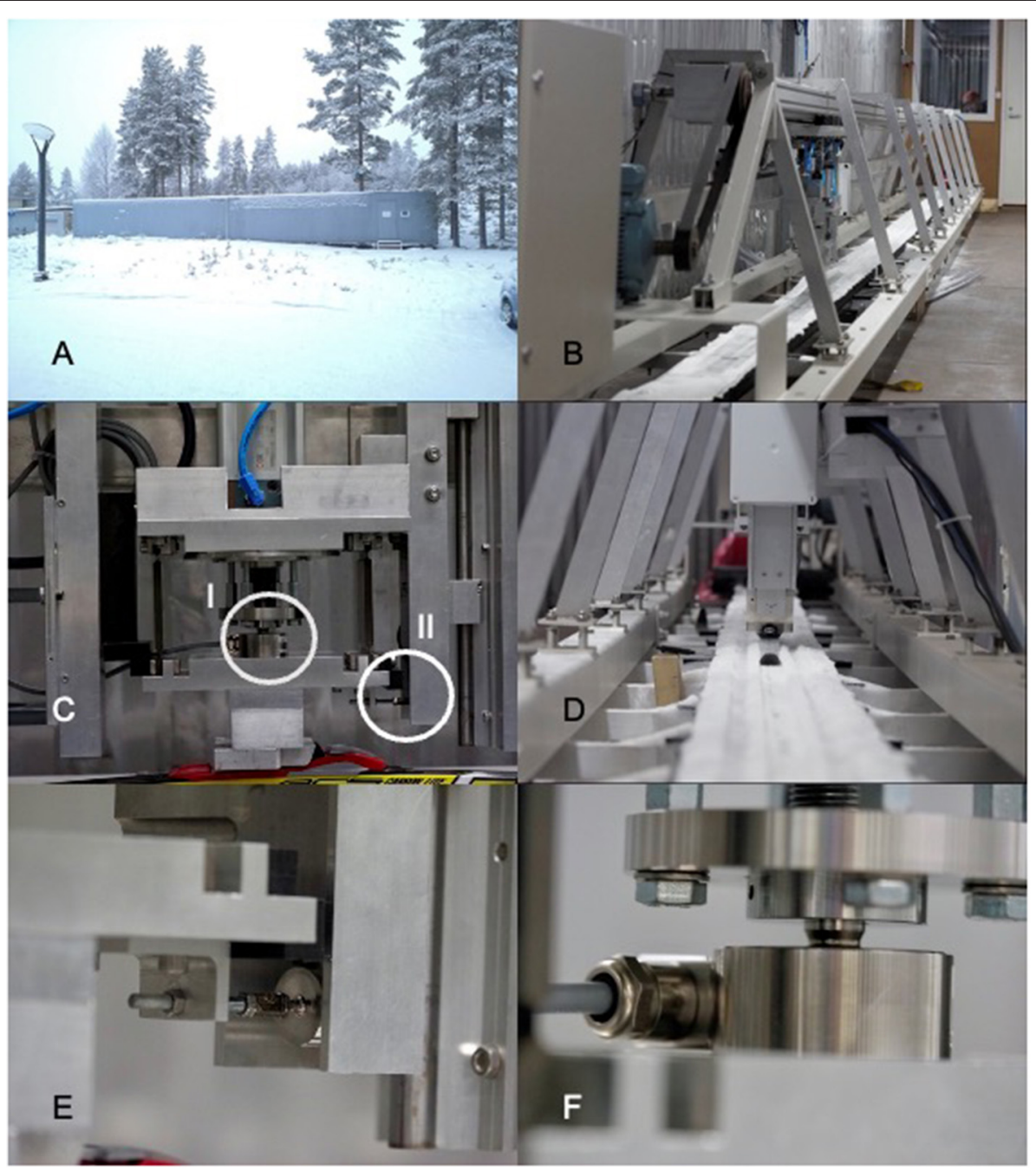

FIGURE 1 | (A) The laboratory is built of two $12 \mathrm{~m}$ long and $2.4 \mathrm{~m}$ wide insulated containers forming a $24 \mathrm{~m}$ long working space. The main section ( $21 \mathrm{~m}$ ) is used as a low temperature laboratory and houses the skitester. A smaller $(3 \mathrm{~m})$ room at the end of the laboratory is used as an entrance and testing office/control room. (B) Frame of the skitester. The frame is $13.7 \mathrm{~m}$ long with an operating length of $10.81 \mathrm{~m}$. Temperature can be set separately for the air and track (C) The skitester measurement unit has separate force sensors to measure vertical (I) and horizontal (II) forces. (D) Ski gliding on the testing track. (E) Closeup picture of horizontal sensor. Same as C(II). (F) Close up picture of vertical sensor. Same as C(I).

temperatures ranging from -20 to $+10^{\circ} \mathrm{C}$. The air cooler has also has a separate warming system that can be programmed to $+5^{\circ} \mathrm{C}$.

The skitester is $13.7 \mathrm{~m}$ long (Figure 1B) and consists of a metal frame and linear actuator (HepcoMotion, DLS5, UK). The ski sledge (Figures 1C,D) can be operated over a distance of $10.81 \mathrm{~m}$. The ski sledge is moved along the linear actuator with an electric motor (Strömberg, HXUR, $7.5 \mathrm{~kW} / 25 \mathrm{Nm}$, Finland). The electric motor is located at the end of the frame where sledge acceleration is started. The motor is controlled with a frequency converter (ABB, ACS800, Sweden).

The pressure applied to the ski in the ski sledge is operated with a pneumatic cylinder $(0-5 \mathrm{kN})$ (Pimatic, P2520, Finland). This cylinder is located on the ski sledge, just above the ski attachment shoe. The pneumatic cylinder is controlled with a pressure regulator (Bosch Rexroth, ED05, Germany). The location of the ski sledge on the frame is determined using an incremental encoder (Sick Stegmann, DRS60, Germany) and the location can be defined at an accuracy of $0.078 \mathrm{~mm}$. The ski attachment shoe is affixed to the sledge with springs that allow for horizontal movement. This, in turn, allows for the loading of the horizontal force sensor.

The force measurement unit is located in the ski sledge. It has separate force sensors for vertical $\left(F_{V}, \pm 2.0 \mathrm{kN}\right)(\mathrm{HBM}, \mathrm{C} 2$, Germany) (Figure 1F) and horizontal $\left(\mathrm{F}_{\mathrm{h}}, \pm 0.5 \mathrm{kN}\right)(\mathrm{HBM}, \mathrm{U} 9 \mathrm{C}$, Germany) forces (Figure 1E). The horizontal sensor is located on the sledge just in front of the ski attachment shoe. The vertical sensor is attached to the top of ski attachment shoe (Figure 1C). Information is transferred to a decoder via wires. The decoder (National Instruments, NI-9205, TX, USA) is read using $1 \mathrm{kHz}$ with custom made computer software (LabView, National Instruments, USA) that is also used for controlling the machine. Calibration of the force measurement unit can be 

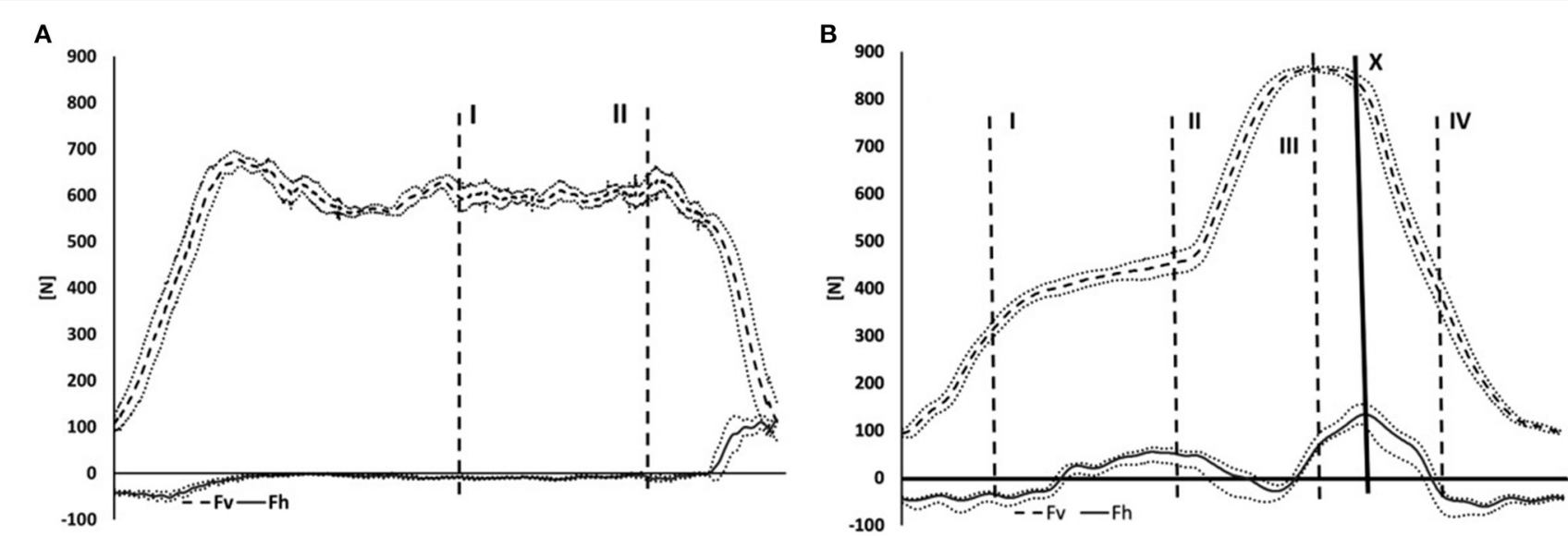

FIGURE 2 | (A) GF is calculated from the steady state (I-II) of the trial with acceleration and braking phases excluded. (B) Three phases of the simulated kick: Gliding phase (I-II), preloading phase (II-III), and kick phase (III-IV). KF is calculated from point of maximal Fh (X). Force curves are presented as average forces of one testing set including standard deviations. On the $x$-axis is one $100 \%$ of measured run and kick.

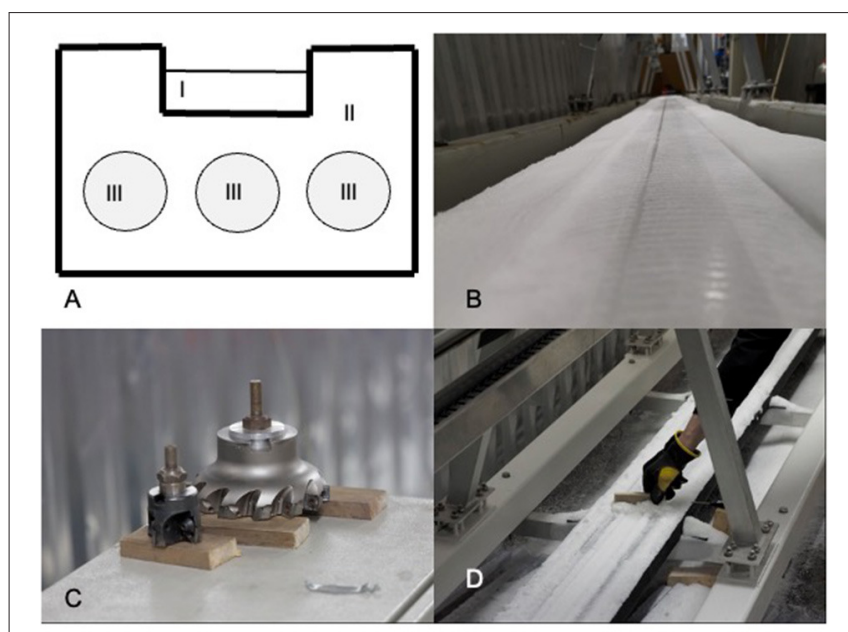

FIGURE 3 | (A) Cross-section of the track. (I) Snow is added to the ice track. (II) Ice accumulates on the track as humidity from the low temperature laboratory freezes on and around the cooling pipes (III) Cooling pipes keep the track frozen. (B) A groove is cut into the ice of the track. (C) Special tools for cutting the groove into the track. (D) Adding of the snow is competed manually.

completed with a custom calibration frame. For measurement of $F_{h}$, the calibration frame is attached to the skitester frame in the beginning of the track. The horizontal force sensor is loaded using a rope, that pulls the ski attachment shoe horizontally backwards. Vertical force sensor calibration is completed in the calibration frame. The vertical force sensor is removed from the sledge and the sensor is loaded by placing weights on the sensor.

\section{Glide Testing}

For each trial, a pneumatic cylinder applies force to the ski while an electric motor simultaneously accelerates the sledge to the desired velocity. Velocity is limited by the software to $6 \mathrm{~m} / \mathrm{s}$. For measurement of gliding friction (GF), a specific measurement area on the ski tester can be defined by the software to ensure that forces are measured from a specific velocity (Figure 2A). GF is calculated as an average value of Fh and Fv (Figure 2A (I-II)) over measurement area (Equation 1).

$$
\frac{F h}{F v}=G F \text { or } K F
$$

Equation 1. Calculation of GF and KF values.

\section{Kick Testing}

To measure the kick friction (KF), forces from a natural classic kick have been simulated with the skitester (Vähäsöyrinki et al., 2008). A single kick consists three phases (Figure 2B). (I-II) The first phase is the gliding phase on one ski. In this phase $50 \%$ of the desired skier mass is applied as vertical force. (II-III) The second phase is the preloading phase where the ski is stopped, and 150\% of the skier mass is applied as vertical force to the ski. (III-IV) The third phase is the kick phase. In the kick phase, the sledge is moved $2 \mathrm{~cm}$ backwards and vertical pressure is released. After the kick phase, the simulation continues with a short swing phase where the ski is moved forward with no force applied to start a new kick simulation. KF is calculated for each simulated kick. The software automatically detects the highest peak of $F_{h}$ from the third phase and KF is calculated from the peak horizontal force by dividing with corresponding vertical force (Equation 1). The skitester runs ten (10) separate simulated kicks for each trial. The result of each trial is reported as an average value of the 10 separate kick simulations.

\section{Track Preparation}

Track preparation is completed in two parts. (1) Before use of the skitester, the track needs to be frozen using the skitester track cooling system. This happens automatically if the cooling system is kept on in humid weather as humidity condenses and freezes over the cooling pipes (Figure 3A). Freezing can be 
completed more quickly by pouring water on the track several times. After the track is frozen and the cooling pipes are covered with a layer of ice, a groove for the ski is cut into the track with special tools that are attached to the ski sledge (Figures 3B,C). (2) Next, snow can be added (Figure 3A (I)) to the track manually (Figure 3D). The small amount of snow needed enables for a quick change of snow and the use of different types of snow. In the present study, snow was made with a high-pressure snow cannon (Ratnik, NY, USA) inside the Vuokatti ski tunnel located next to the laboratory. Snow was collected from the tunnel and moved to the low temperature laboratory in a plastic container. Snow was stored and used for a maximum of $12 \mathrm{~h}$ in stable laboratory conditions.

Renewing the snow on the track requires two steps. (1) Old snow is removed; (2) New snow is applied to the track manually (Figure 3D). New snow was packed 10 times using a separate ski and was completed with the same setup and protocol $(0.6 \mathrm{kN}$ and $4 \mathrm{~m} / \mathrm{s}$ ) as used in glide testing. During kick testing, the track was packed between each run by performing five glide tests before a new kick test. Extra packing is needed in kick testing because the track is exposed to stress and, depending on snow type, snow may shift during each kick.

\section{Measurement Protocol}

\section{Environment Control}

Air temperature and humidity were monitored throughout the present study with a device (Vaisala, HM40, Finland) located in the middle of the skitester frame at the height of $1 \mathrm{~m}$. Snow temperature (Swix, T93, Norway) was measured from the middle of the track. Snow free water content (Snow moisture meter hydrogen 011, Doser Messtechnic, Kempten, Germany) was assessed from the same spot. Snow free water values must be considered carefully because of the possible effect of the ice under the thin snow layer. Snow density $\left(672.4 \mathrm{~kg} / \mathrm{m}^{2}\right)$ was analyzed from each container collected from the ski tunnel. Snow density was calculated as an average value of 5 measurements. Grain size $(0.78 \mathrm{~mm} \pm 0.38)$ was analyzed visually from macroscopic pictures using 100 representative snow crystals. Size was analyzed using picture manipulation software (GIMP, www.gimp.org).

\section{Ski Preparation}

Two cross-country skating skis were used for all GF tests (Madshus redline, $191 \mathrm{~cm}$, Norway). Skis were freshly ground (Swecom, Italy). Roughness of the ski base was measured with a profilometer (Mitutoyo Surftest SJ-31, Japan). Stiffness of each ski was measured using the Finish National Ski Team's standard protocol using a Classic Ski Control device (Tuotepalvelu Isohannu Oy, Ylöjärvi, Finland, Table 1). Two different skis (A and $\mathrm{B}$ ) were used during the glide tests. All precision tests were done with ski A while pressure and velocity were tested with ski B (Table 1). KF testing was competed with a skin-grip ski (Salomon S-lab/skin, $201 \mathrm{~cm}$, France). After each measured set, the ski base was refreshed by brushing the base with a fine metal brush (RedCreek, Sweden). After brushing, a fresh layer of liquid glide wax (LF Polar, Vauhti speed Oy, Joensuu, Finland) was applied to the base. After the liquid wax was dried, the base of
TABLE 1 | Properties measured from measured skis.

\begin{tabular}{lccc}
\hline Ski & Roughness $[\mu \mathbf{m}]$ & Ski height $[\mathbf{m m}]$ & Stiffness [kg] \\
\hline Madshus redline skate A & 3.3 & 3.1 & 90.0 \\
Madshus redline skate B & 3.3 & 3.0 & 85.0 \\
Salomon S-lab classic & 3.5 & 1.6 & 54.0 \\
\hline
\end{tabular}

Ski base average roughness, ski height, and ski stiffness. Ski height and stiffness were measured using standard Finish Ski Team protocols.

TABLE 2 | Programmed laboratory temperature settings for three different conditions: WARM, MEDIUM, and COLD.

\begin{tabular}{lcc}
\hline Temperature setting & Air $\left[{ }^{\circ} \mathbf{C}\right]$ & Track $\left[{ }^{\circ} \mathbf{C}\right]$ \\
\hline WARM & -1 & -1 \\
MEDIUM & -6 & -6 \\
COLD & -12 & -12 \\
\hline
\end{tabular}

TABLE 3 | Measurement protocols.

\begin{tabular}{llcccl}
\hline Measurement & Condition & Protocol & $\begin{array}{c}\text { Velocity } \\
{[\mathbf{m} / \mathbf{s}]}\end{array}$ & $\begin{array}{c}\text { Pressure } \\
{[\mathbf{N}]}\end{array}$ & Ski \\
\hline Precision & WARM & $5 \times 50$ & 4 & 600 & Skate A \\
Precision & MEDIUM & $5 \times 50$ & 4 & 600 & Skate A \\
Precision & COLD & $5 \times 50$ & 4 & 600 & Skate A \\
Pressure & MEDIUM & $3 \times 50$ & 4 & 600,900, & Skate B \\
& & & & 1200 & \\
Velocity & MEDIUM & $3 \times 50$ & $2,4,6$ & 600 & Skate B \\
Kick test & MEDIUM & $5 \times 10$ & - & $800 *$ & Classic/Skin
\end{tabular}

Six different measurements were carried out in the study. Precision measurements were carried out in different conditions. For pressure, velocity, and kick measurements MEDIUM condition was used. Protocol, velocity, and pressure changed between measurements. *In kick testing pressure of $800 \mathrm{~N}$ was used to calculate the desired pressures for three phases (gliding, preloading, and kick) of the test. Three different skis were used during the measurements.

${ }^{*}$ Pressure used in simulation.

the ski was brushed gently using a fine metal brush (RedCreek, Sweden) and finished with a nylon brush (RedCreek, Sweden).

\section{Precision and Repeatability in Glide Testing}

Precision testing was completed in three different conditions: WARM, MEDIUM, and COLD (Table 2). In all precision tests the same pressure and velocity were used $(0.6 \mathrm{kN}$ and $4 \mathrm{~m} / \mathrm{s})$. The test protocol consisted of five sets of $50(i=1-50)$ glides in each condition (Table 3 ). After each set of 50 glides, used snow was removed from the track and replaced such that the track was prepared five times for each condition. The goal of preparing the track between each set was to maintain the track condition.

In all conditions (1) overall precision, (2) precision of the measurement unit, and (3) the influence of the snow and ski was analyzed (Table 4). Precision calculations were completed based on Hasler et al. (2016). Because GF values are changing nonlinearly during the first 10 runs, the later runs $(i=11-50)$ were used for precision calculations. 
TABLE 4 | Calculation of precision values.

\begin{tabular}{|c|c|c|c|}
\hline Precision value & Equation & Note & Relative SD \\
\hline Overall precision & $\varepsilon_{i}=\sqrt{\frac{1}{5} \sum_{j=1}^{5}\left(\mu_{j, i}-\bar{\mu}_{i}\right)^{2}}$ & Where $\mu_{j, i}$ is GF $\mathrm{i}$ value of $\mathrm{j}$ test and $\bar{\mu}_{i}$ is the mean of five $\mathrm{i}$ values & $=\varepsilon_{i} / \bar{\mu}_{i}$ \\
\hline Precision of the measurement unit & $\varepsilon_{m u}=\sqrt{\sum_{i=11}^{50} \frac{1}{40}\left(\mu_{i}-\mu_{i \text { fit }}\right)^{2}}$ & Where $\mu_{i}$ is GF value of $\mathrm{i}$ and $\mu_{i}$ fit is corresponding value i from linear regression fit & $=\varepsilon_{m u} / \bar{\mu}$ \\
\hline Influence of the ski and snow & $\varepsilon_{S S, i}=\sqrt{\sum_{j=1}^{5} \frac{1}{5}\left(\mu_{j, i}-\bar{\mu}_{i \text { fit }}\right)^{2}}$ & Where $\mu_{j, i}$ is ith linear regression fit and $\bar{\mu}_{i}$ fit is the mean of linear fit of j:th test & $=\varepsilon_{S S, i} / \mathrm{i}\left(\mu_{i}\right.$ fit $)$ \\
\hline
\end{tabular}

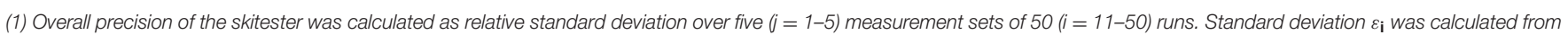

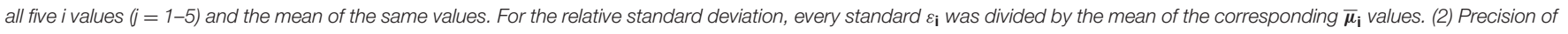

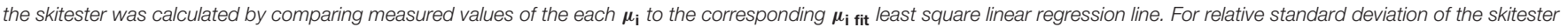

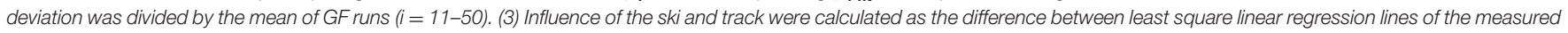
five sets. Standard deviation of linear fit lines were calculated in each $\varepsilon_{\mathbf{s s}, \mathbf{i}}$. For relative standard deviation, standard deviations were related to the mean values of linear fit $i$ values.

\section{Effect of Pressure and Velocity}

Pressure testing was performed one test at a time. Three different pressure settings were used $(0.6 \mathrm{kN}, 0.9 \mathrm{kN}$, and $1.2 \mathrm{kN})$ utilizing forces that normally occur in both classic and skate skiing (Leppävuori et al., 1993; Vähäsöyrinki et al., 2008). Velocity testing was performed at three different speeds $(2,4$, and 6 $\mathrm{m} / \mathrm{s}$ ). Both pressure and velocity measurement were analyzed in each 50 sets as least square regression lines over $i=11-50$ to show changes in GF levels and in measurement unit precision (Table 3). The track was prepared according to the previously described protocols prior to each set of velocity and pressure tests.

\section{Precision and Repeatability of KF Testing}

The KF test consisted of five sets of 10 repetitions $(i=1-10)$ that were averaged for analysis. After each set of 10 tests, the snow was changed and the track prepared according to the previously described protocol. Precision of KF tests were analyzed using the previously described methods.

\section{RESULTS}

Average temperatures for the air and track remained close to the desired values although larger variation was observed in WARM condition $(S D \pm 1.6)$ while MEDIUM (SD \pm 0.6$)$, and COLD ( $\mathrm{SD} \pm 0.4$ ) conditions remained more stable. MEDIUM $\left(-6.3^{\circ} \mathrm{C}\right)$ temperature conditions showed meaningful variation when same temperature was desired for pressure $\left(-7.7^{\circ} \mathrm{C}\right)$, velocity $\left(-5.7^{\circ} \mathrm{C}\right)$, and kick $\left(-8.7^{\circ} \mathrm{C}\right)$ tests. Only in COLD was the temperature higher $\left(-8.3\right.$ vs. $\left.-12^{\circ} \mathrm{C}\right)$ than the temperature setting for the laboratory (Table 2).

\section{Precession of the Skitester and Influence of the Condition}

In all glide test sets, the change in GF was greatest over the first 10 glides $(i=1-10)$ and was more stable in glides 1150 (Figure 4D). Non-linear changes in GF values were greatest during WARM conditions. Least square linear regression lines showed variation after each track preparation while GF values also showed meaningful variation. The tendencies in GF where mainly positive, but in MEDIUM and WARM conditions testing sets were also negative meaning that the glide improved during the set (Figures 4A-C).
Overall precision of the skitester showed some variation in different conditions, however, precision values stabilized after the first 10 glides and remained more stable after that. Overall precision $(i=11-50)$ in the MEDIUM condition showed the lowest relative variation values $(2.4 \%)$. In the COLD condition, relative variation was slightly higher $(3.0 \%)$ and in the WARM condition (5.5\%) relative variation was highest. Precision of the measurement unit stayed under $1 \%$ in the COLD and MEDIUM conditions. In the WARM condition, the measurement unit performed slightly worse (COLD 0.6\%, MEDIUM 0.8\%, WARM $1.1 \%)$. The influence of the ski and track showed the highest relative variation of the analyzed measures. The highest variation was observed in the COLD condition (16.7\%) and lowest in the MEDIUM (10.4\%) condition (Table 5). Influence of ski and snow changed in different conditions. In the WARM condition, the influence of the ski and snow increased toward the end of the set. In MEDIUM and COLD conditions, the influence of ski and snow decreased toward the end of the set (Figure 4E).

\section{Effect of Velocity and Pressure}

GF values had a tendency to shift from slightly negative to positive when velocity was increased from 4 to $6 \mathrm{~m} / \mathrm{s}$ (Figure 5A). In the pressure test, slightly greater GF values were observed when pressure increased (Figure 5B). Measurement unit precision was lowest at $4 \mathrm{~m} / \mathrm{s}$ and was slightly worse in other examined velocities [1.16\% $(2 \mathrm{~m} / \mathrm{s}), 0.86 \%(4 \mathrm{~m} / \mathrm{s})$, and $1.31 \%$ $(6 \mathrm{~m} / \mathrm{s})]$. In the pressure test, measurement unit precision was lowest at $0.6 \mathrm{kN}(0.69 \%)$ and $0.9 \mathrm{kN}(0.72 \%)$ and higher at 1.2 $\mathrm{kN}(2.06 \%)$.

\section{KF Measurement}

Precision calculations for KF testing were completed for $i=1-$ 10 (Figure 6A). Overall precision and skitester precision with regard to variation in kick testing was similar (2.5 vs. $2.4 \%$ ) (Table 5). The influence of ski and snow was $4.1 \%$. The effect of ski and snow decreased slightly from 4.4 to $3.9 \%$ in test seven after which the influence of the snow increased to $4.05 \%$ by test 10 (Figure 6B).

\section{DISCUSSION}

\section{Main Results}

In glide testing the skitester showed precision between 0.6 and $1.1 \%$. In overall precision, where a shift in GF values during 

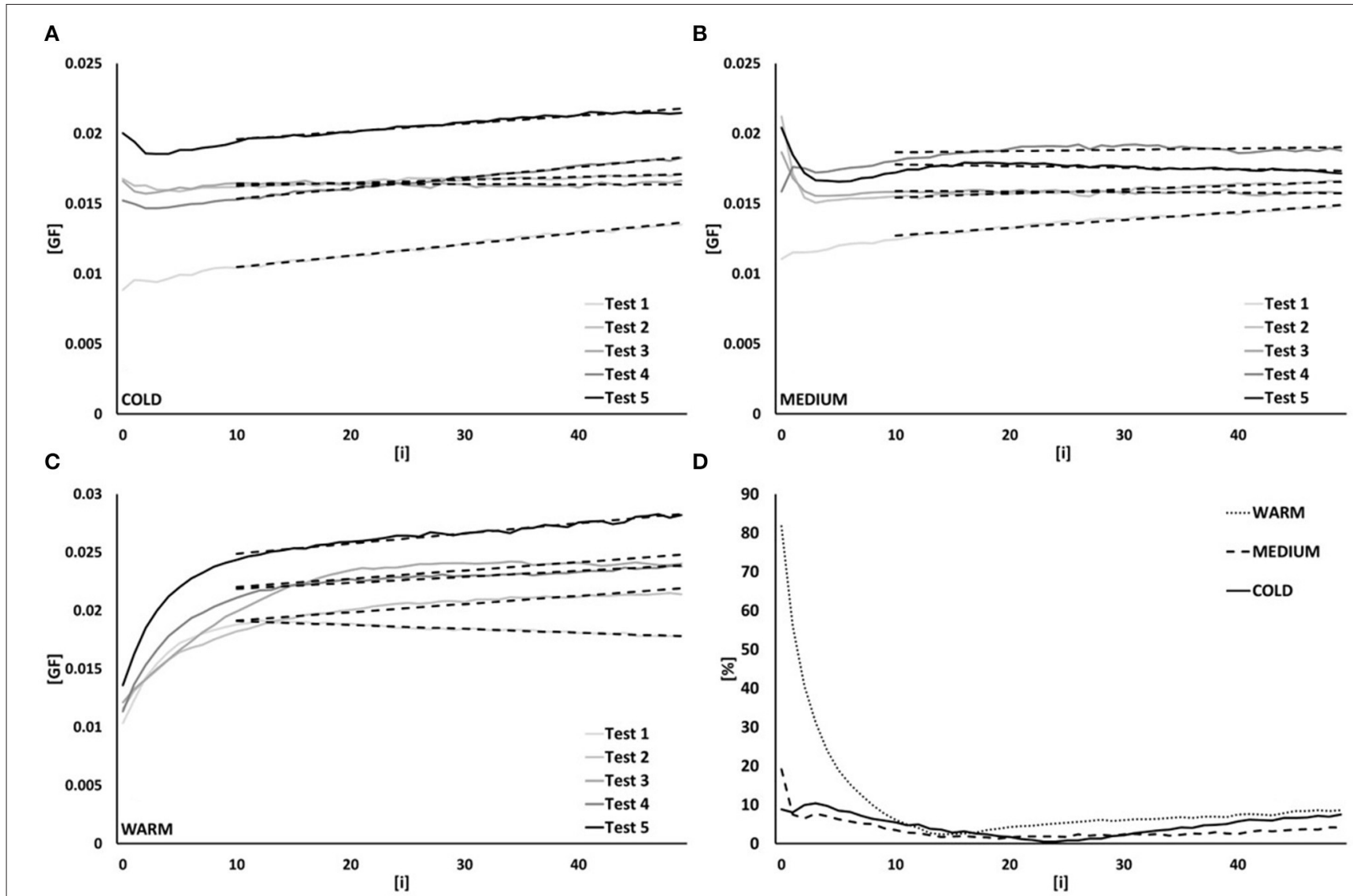

E

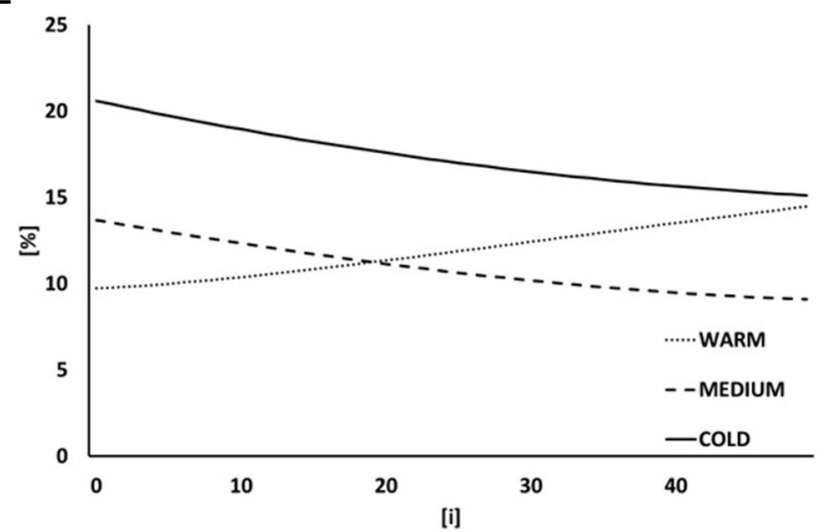

FIGURE 4 | Measured values of five tests of 50 runs and linear regression lines over $i=11-50$ runs where (A) COLD, (B) MEDIUM, and (C) WARM conditions. (D) Overall precision values in different condition (COLD, MEDIUM, and WARM) during measurements $(i=1-50)$. (E) Effect of snow and ski in different conditions [COLD, MEDIUM, and WARM during measurements $(i=1-50)]$. In all $(\mathbf{A}-\mathbf{E}) \mathrm{x}$-axis i:th value of the set. In $(\mathbf{A}-\mathbf{C})$ figures $y$-axis is measured $G F$ values. (D,E) $y$-axis indicates relative variation.

measurement was taken into account, variation was higher 3.0$5.5 \%$. The influence of the ski and snow demonstrated the most variation in calculated precision values $10.4-16.7 \%$. Based on these results, the skitester is reliable for glide testing during one track preparation and the observed shift in GF values needs to be taken into an account when using the skitester. The influence of the ski and snow on measurement precision was greater than expected, which suggests that ski and track preparation should be further developed.

In kick testing, variation in overall precision and measurement unit precision were at the same level meaning that the shift in $\mathrm{KF}$ values and measurement unit precision have equal influence on the measurement. The influence of ski and snow showed greater variation, but it was significantly lower compared to glide testing 

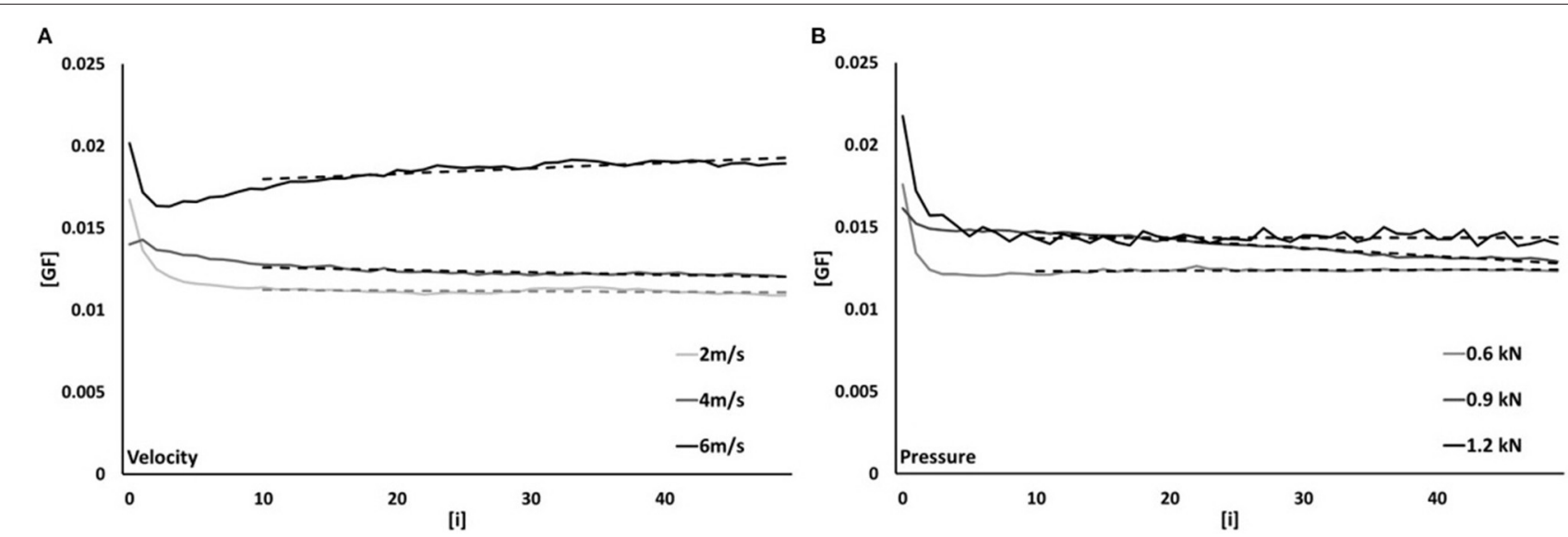

FIGURE 5 | GF values $(i=1-50)$ and linear regression lines $(i=11-50)$. (A) Effect of velocity $(2,4$, and $6 \mathrm{~m} / \mathrm{s})$ and (B) effect of pressure $(0.6,0.9$, and $1.2 \mathrm{kN})$.

TABLE 5 | Overall precision, precision of the skitester, and influence of ski and snow.

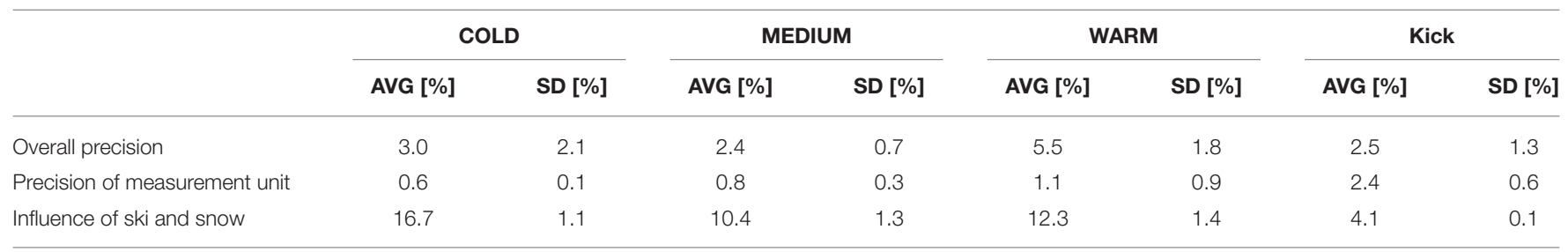

Relative standard deviation values were taken as precision values (AVG). Standard deviations were calculated over different measurement sets (overall precision and precision of the ski tester) or over $i=11-50$ (Influence of ski and snow) in different conditions and kick testing.
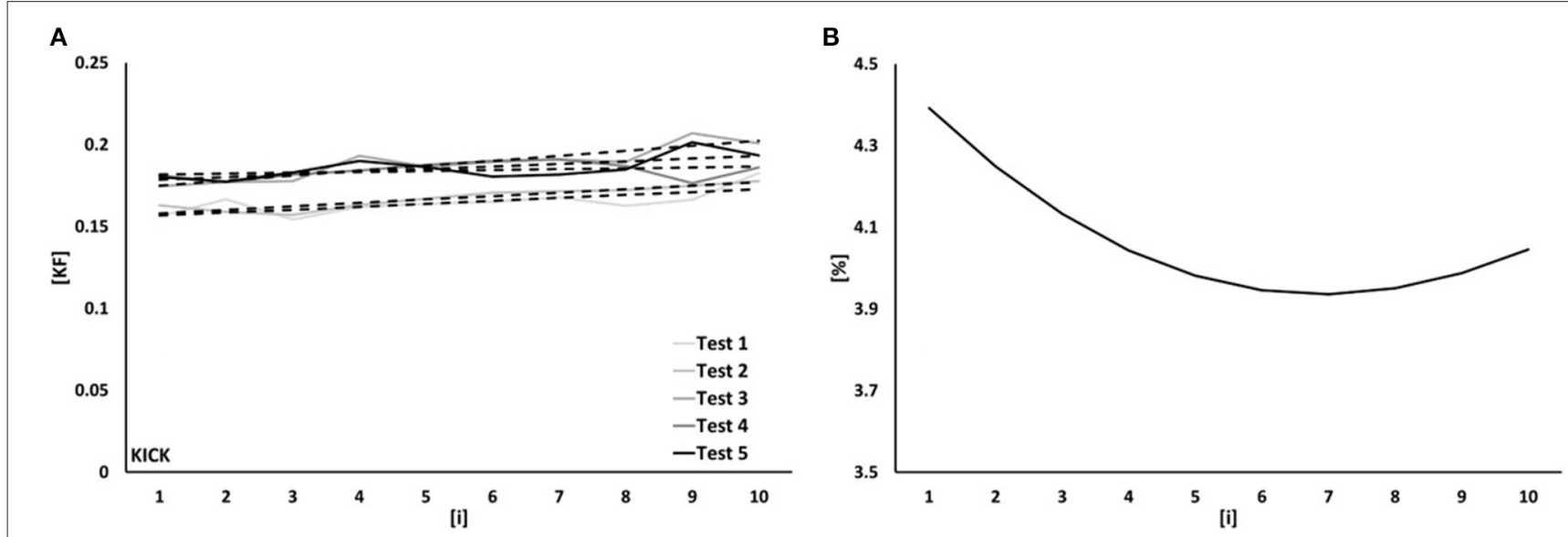

FIGURE 6 | (A) KF values and linear regressions in five tests series over $i=1-10$ the measurement set.

[10.4\% (MEDIUM) vs. 4.1\%]. There is no earlier data to evaluate the effect of this variation, in practice. Nevertheless, to the best of our knowledge, the skitester offers reasonable precision to identify similar characteristics in individual classic skis.

\section{Environmental Management System}

Generally, stable conditions were harder to maintain for the WARM condition (Tables 2,6 ) whereas COLD conditions were easier to maintain inside the laboratory. This is mainly due to the cooling system, which runs at higher frequency, meaning that system runs more often and for longer periods when the desired temperature is colder. In WARM $\left(-1^{\circ} \mathrm{C}\right)$ conditions, the cooling system runs at lower frequency causing more variation in air temperature. Already in MEDIUM $\left(-6^{\circ} \mathrm{C}\right)$ condition cooling ran more frequently compared to WARM, which was observed from reported SD (1.6 vs. $0.6 \%)$ values 
TABLE 6 | Snow and air conditions during measurements.

\begin{tabular}{|c|c|c|c|c|c|c|c|c|c|c|c|c|}
\hline & \multicolumn{2}{|c|}{ WARM } & \multicolumn{2}{|c|}{ MEDIUM } & \multicolumn{2}{|c|}{ COLD } & \multicolumn{2}{|c|}{ Pressure } & \multicolumn{2}{|c|}{ Velocity } & \multicolumn{2}{|c|}{ Kick } \\
\hline & AVG & SD & AVG & SD & AVG & SD & AVG & SD & AVG & SD & AVG & SD \\
\hline Air temperature $\left[{ }^{\circ} \mathrm{C}\right]$ & -0.3 & 1.6 & -6.3 & 0.6 & -8.3 & 0.4 & -7.7 & 1.3 & -5.7 & 0.1 & -8.7 & 1.1 \\
\hline Relative humidity [\%] & 64.7 & 6.2 & 57.4 & 2.5 & 46.7 & 2.7 & 54.1 & 3.2 & 54.5 & 0.2 & 52.2 & 1.3 \\
\hline Snow temperature $\left[{ }^{\circ} \mathrm{C}\right]$ & -2.4 & 0.5 & -7.3 & 0.5 & -13.9 & 0.2 & -7.8 & 0.3 & -7.8 & 0.4 & -8.1 & 0.3 \\
\hline Doser [F] & 38.2 & 1.3 & 39.8 & 1.0 & 37.4 & 0.5 & 40.5 & 0.9 & 40.3 & 0.5 & 40.8 & 0.7 \\
\hline
\end{tabular}

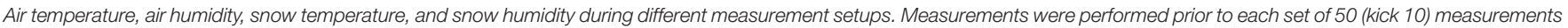
and the average (AVG) and standard deviation (SD) values were calculated over the measurement in each setup (WARM, MEDIUM, COLD, pressure, velocity, and kick).

of the temperature measurement. Limitations in the laboratory cooling system are related to the small air space. Changes caused by the cooling system, or just by entering the cold laboratory, can affect the overall temperature and temperature distribution. These changes must be minimized to achieve more stable measurement/testing results.

Snow deformation occurs in different conditions and tests at different pressures and velocities (Eriksson and Nupen, 1955). Snow deformation was observed in the present study as the testing track started to shine during measurement sets. The change in GF was greater at lower temperatures and at higher velocities. Pressure did not clearly change the GF tendency. Interestingly, the shift in GF values did not have a constant direction. Tendencies in individual tests sets was negative or positive within the test setup. In MEDIDUM, the direction of the change varied between track preparations. Possible explanations for this observation include variation in snow quality or ski preparation. Another possibility is that the track preparation process is inadequate. A perfectly even track is very difficult to achieve and even small differences in track preparation can affect the ski snow contact area and snow deformation. Vibration from the frame of the skitester may also influence the measurement (Koptyug et al., 2012). However, this influence is probably greater for the glide testing because measured forces are smaller.

\section{GF Measurement}

GF testing precision values varied according to the condition. In comparison to previously reported precision values by Hasler et al. (2016), overall precision in MEDIUM was $2.4 \%$, which is $1 \%$ higher than previously reported (1.4). Precision of the measurement unit showed similar relative variation $[0.8 \%$ MEDIUM vs. $0.4 \%(3 \mathrm{~m} / \mathrm{s}), 1.2 \%(10 \mathrm{~m} / \mathrm{s})]$ as previously reported. Nevertheless, track preparation and snow deformation were a greater source of variation and were clearly higher than in earlier reports (10.4\% medium vs. $1.2-2.2 \%$ ).

Measurements were carried out in three different conditions. Actual GF values were similar between MEDIUM and COLD conditions with an average of 0.016. In WARM, GF average was higher at 0.021 . Also, tendencies during the measurement where more similar between COLD and MEDIUM than between WARM and MEDIUM, which suggests that MEDIUM and COLD conditions are similar with used snow. In the future, more aggressive snow and/or colder temperatures will be needed to achieve more meaningful differences.

\section{Velocity and Pressure Measurement}

The values for GF increased with velocity, in line with study of Hasler et al. (2016). This result is also in line with the observed increase in CoF with velocity (Moldestad, 1999, 13). Measurement unit precision did not show a linear decrease in precision, but the lowest value $(0.86 \%)$ was observed at $4 \mathrm{~m} / \mathrm{s}$. In pressure testing, the measured GF values were slightly higher with higher pressure. The most meaningful differences observed were in the precision of the measurement unit where variation ranged from 0.72 to $2.06 \%$ when pressure was changed from 0.9 to $1.2 \mathrm{kN}$. An increase in variation can be caused by the bending in the structures under the track or the increased vibrations of the measurement sledge when high pressure is applied.

\section{KF Measurement}

No previous comparison was found for kick testing precision values. Although the precision of the measurement unit was somewhat greater in the glide test, the kick test involves greater forces and differences between measurable variables are often greater. Moreover, the ski preparation process for kick testing includes more variables such as achieving an exact thickness in the applied layers of grip wax. Overall, the precision of kick testing is challenging to achieve. During the kick phase, great stress is applied to the track and changes inside the snow layer under the ski can influence the measured forces. In the current test a skin ski was used to ensure minimal change in kick areas properties of the test ski. In the future the kick testing can be combined with glide testing to achieve a better overall picture of the classic ski performance.

\section{Limitations}

Renewing of the snow on the track is relatively fast process. Unfortunately, maintaining the desired friction conditions appears to be challenging. In the present investigation, the influence of the ski and snow showed values over $10 \%$, which are rather high if differences between 1 and $2 \%$ are desired and previously reported (Hasler et al., 2016). With the current skitester model, these limitations suggest that results are not comparable between different track preparations. Other possible sources of error in glide testing come from ski preparation. The test ski was waxed with liquid wax in the cold environment and wax was added for every preparation. The ski preparation process needs to be re-evaluated and compared to e.g., just cleaning or brushing the ski between tests. 
In the coming development of the skitester, the factors mentioned above must be considered. Better control over the processes for preparing the snow and track is essential to achieve better precision. Outside weather may affect the relative humidity and temperature stability of the current version of the cold laboratory. During the present measurements, the conditions outside were stable and typical of autumn weather in the Vuokatti area with an average temperature $5.3^{\circ} \mathrm{C}$ and relative humidity of $80.7 \%$. Controlling of the laboratory temperature and humidity would be easier in a larger space.

\section{Conclusions}

Measurement of glide from cross-country skis is a complicated process even when completed in laboratory conditions. Total control of all influencing factors from the testing environment as well as in the utilized equipment is challenging and needs to be completed with care. Laboratory measurements provide the advantages of being able to test skis while excluding factors such as weather and the influence of the ski service members and dependence of testing skis in pairs. Several practical implications

\section{REFERENCES}

Ainegren, M., Carlsson, P., Laaksonen, M. S., and Tinnsten, M. (2014). The influence of grip on oxygen consumption and leg forces when using classical style roller skis. Scand. J. Med. Sci. Sports 24, 301-310. doi: 10.1111/sms.12006

Bäckström, M., Dahlen, L., and Tinnsten, M. (2008). "Essential ski characteristics for cross-country skis performance," in InISEA 2008 Conference on Engineering of Sport 7 (Biarritz: Springer), 543-549.

Bäurle, L. (2006). Sliding friction of polyethylene on snow and ice (Doctoral thesis). ETH Zürich, Zürich, Switzerland.

Breitschädel, F., Haaland, N., and Espallargas, N. (2014). A tribological study of UHMWPE ski base treated with nano ski wax and its effects and benefits on performance. Proc. Eng. 72, 267-272. doi: 10.1016/j.proeng.2014.06.048

Breitschädel, F., Klein-Paste, A., and Løset, S. (2010a). Effects of temperature change on cross-country ski characteristics. Proc. Eng. 2, 2913-2918. doi: $10.1016 /$ j.proeng.2010.04.087

Breitschädel, F., Lund, Ø., and Løset, S. (2010b). Cross country ski base tuning with structure imprint tools. Proc. Eng. 2, 2907-2911. doi: 10.1016/j.proeng.2010.04.086

Buhl, D., Fauve, M., and Rhyner, H. (2001). The kinetic friction of polyethylen on snow: the influence of the snow temperature and the load. Cold Reg. Sci. Technol. 33, 133-140. doi: 10.1016/S0165-232X(01)00034-9

Colbeck, S. C. (1994). A review of the friction of snow skis. J. Sports Sci. 12, 285-295. doi: 10.1080/02640419408732174

Eriksson, R., and Nupen, W. (1955). Friction of Runners on Snow and Ice. Hanover, $\mathrm{NH}$ : Cold Regions Research and Engineering Lab.

Fauve, M., Buhl, D., Rhyner, H. U., Schneebeli, M., and Ammann, W. (2005), "Influence of snow and weather characteristics on the gliding properties of skis," in Science and Skiing III (Aspen, CO), 401-410.

Fenre, M. D. (2016). Development of a Linear Tribometer. Trondheim: NTNU.

Hamrock, B. J., and Dowson, D. (1981). Ball Bearing Lubrication: The Elastohydrodynamics of Elliptical Contacts. https://www.google.com/search? sxsrf=ALeKk02hxA6MaLpt7_0Qw4a31VgtX8UKNA:1618896037884\&q= Hoboken\&stick=H4sIAAAAAAAAAOPgE-LUz9U3MMotLypT4gAxK_ LMk7S0spOt9POL0hPzMqsSSzLz81A4VhmpiSmFpYlFJalFxYtY2T3yk_ KzU_N2sDICAC-vG35QAAAA\&sa=X\&ved=

2ahUKEwjooZDaiYzwAhUzzTgGHRjzBXYQmxMoATAfegQIMRAD Hoboken, NJ: Wiley.

Hasler, M., Schindelwig, K., Mayr, B., Knoflach, C.h., Rohm, S., van Putten, J., et al. (2016). A novel ski-snow tribometer and its precision. Tribol. Lett. 63:33. doi: $10.1007 /$ s11249-016-0719-2 have been developed for the current skitester. The primary function of the current skitester has been the selection of test skis, both skate and classic. During the selection and pairing process, the skis that demonstrate the most similar characteristics are selected and re-paired. In future work, the relationship of skitester results to testing on the ski track outside will be clarified.

\section{DATA AVAILABILITY STATEMENT}

The raw data supporting the conclusions of this article will be made available by the authors, without undue reservation.

\section{AUTHOR CONTRIBUTIONS}

TL is responsible of data collection, analyzes, desing of the study, and most of the writing. $\mathrm{TH}$ is technical person developing the skitester and carried out the measurements together with TL. OO contribution to study design, writing work, reading, and commenting. SL and VL reading and commenting. All authors contributed to the article and approved the submitted version.

ICR Cross Country (2020). Available online at: https://assets.fis-ski.com/image/ upload/v1608218112/fis-prod/assets/ICR_CrossCountry_2020_clean.pdf (accessed January 5, 2021).

Karlöf, L., and Axell, L. T. (2005). On Dry Lubricants in Ski Waxes. Technical Note, 4. Lillehammer: Swix AS.

Koptyug, A., Bäckström, M., Tinnsten, M., and Carlsson, P. (2012). Cross-country ski vibrations and possible mechanisms of their influence on the free gliding. Proc. Eng. 34, 473-478. doi: 10.1016/j.proeng.2012.04.081

Leppävuori, A. P., Karras, M., Rusko, H., and Viitasaio, J. T. (1993). A new method of measuring 3-D ground reaction forces under the ski during skiing on snow. J. Appl. Biomech. 9, 315-328. doi: 10.1123/jab.9.4.315

Linnamo, V., Kolehmainen, V., Vähäsöyrinki, and Komi, P. (2009a). "Simulation of classical skiing using a new skitester," in Science and Skiing IV, eds E. Müller, S. Lindinger, and T. Stöggl (Oxford: Meyer and Meyer Sport), 615-620.

Linnamo, V., Piirainen, J., Vähäsöyrinki, P., and Komi, P. (2009b). "Quality of grip waxing and the force production in classical skiing," in 14th Annual Congress of the European College of Sport Science (Oslo), 294.

Moldestad, D. A. (1999). Some aspects of ski base sliding friction and ski base structure (Doctoral thesis). NTNU. Trondheim, Norway.

Moxnes, J. F., Sandbakk, Ø., and Hausken, K. (2014). Using the power balance model to simulate cross-country skiing on varying terrain. Open Access J. Sports Med. 2014, 89-98. doi: 10.2147/OAJSM.S53503

Nachbauer, W., Schröcksnadel, P., and Lackinger, B. (1996). "Effects of snow and air conditions on ski friction," in Skiing Trauma and Safety, Vol. 10, eds C. Daniel Mote, R. J. Johnson, W. Hauser, and P. S. Schaff (West Conshohocken, PA: ASTM International), 78-185.

Pellegrini, B., Stöggl, T. L., and Holmberg, H.-C. (2018). Developments in the biomechanics and equipment of olympic cross-country skiers. Front. Physiol. 9:976. doi: 10.3389/fphys.2018.00976

Shimbo, M. (1961). The Mechanism of Sliding on Snow. Union Geodesique et Geophysique Internationale. https://www.google.com/search?sxsrf=ALeKk01UBh7qqrpbkgqMfre_OHNOsI tG3Q:1618897201404\&q= +Oxfordshire\&stick=H4sIAAAAAAAAAOPgE-L Sz9U3MEmuzEk2VOIAsYtyS3K0jDLKrfST83NyUpNLMvPz9POL0hPzMqs SQZxiq4zUxJTC0sSiktSiYoWc_GSw8CJWifDEnJzMvPS0_KIUHQX_ChBdnJ FZlLqDIREAcXb7jWoAAAA\&sa=X\&ved=2ahUKEwiD8feEjozwAhWqILcA HSF8ATEQmxMoATAZegQIHBAD Wallingford: Association Internationale d'Hydrologie Scientifique, 101-06.

Spring, E., Savolainen, S., Erkkilä, J., Hämäläinen, T., and Pihkala, P. (1988). Drag area of a cross-country skier. J. Appl. Biomech. 4, 103-113. doi: $10.1123 /$ ijsb.4.2.103 
Stöggl, T., Ohtonen, O., Takeda, M., Miyamoto, N., Snyder, C., Lemmettyl,ä, T., et al. (2019). Comparison of exclusive double poling to classic techniques of cross-country skiing. Med. Sci. Sports Exerc. 51, 760-772. doi: 10.1249/MSS.0000000000 001840

Swarén, M., Karlöf, L., Holmberg, H.-C., and Eriksson, A. (2014). Validation of test setup to evaluate glide performance in skis. Sports Technol. 7, 89-97. doi: 10.1080/19346182.2014. 968164

Theile, T., Szabo, D., Luthi, A., Rhyner, H., and Schneebeli, M. (2009). Mechanics of the ski-snow contact. Tribol. Lett. 36, 223-231. doi: 10.1007/s11249-009-9476-9

Vähäsöyrinki, P., Komi, P. V., SeppäLä, S., Ishikawa, M., Kolehmainen, V., Salmi, J. A., et al. (2008). Effect of skiing speed on ski and pole forces in cross-country skiing. Med. Sci. Sports Exerc. 40, 1111-1116. doi: 10.1249/MSS.0b013e3181666a 88

Conflict of Interest: The authors declare that the research was conducted in the absence of any commercial or financial relationships that could be construed as a potential conflict of interest.

Copyright (๑) 2021 Lemmettylä, Heikkinen, Ohtonen, Lindinger and Linnamo. This is an open-access article distributed under the terms of the Creative Commons Attribution License (CC BY). The use, distribution or reproduction in other forums is permitted, provided the original author(s) and the copyright owner(s) are credited and that the original publication in this journal is cited, in accordance with accepted academic practice. No use, distribution or reproduction is permitted which does not comply with these terms. 